\title{
An apparent paradox of horizontal and vertical disease transmission
}

\author{
STANLEY H. FAETH $\dagger$, KARL P. HADELER $\ddagger$ and HORST R. THIEME $\ddagger$ \\ $†$ School of Life Sciences, Arizona State University, Tempe, AZ 85287-4501, USA \\ $\ddagger$ Department of Mathematics and Statistics, Arizona State University, Tempe, AZ 85287-1804, USA
}

(Received 17 September 2006; in final form 21 September 2006)

\begin{abstract}
The question as to how the ratio of horizontal to vertical transmission depends on the coefficient of horizontal transmission is investigated in host-parasite models with one or two parasite strains. In an apparent paradox, this ratio decreases as the coefficient is increased provided that the ratio is taken at the equilibrium at which both host and parasite persist. Moreover, a completely vertically transmitted parasite strain that would go extinct on its own can coexist with a more harmful horizontally transmitted strain by protecting the host against it.
\end{abstract}

Keywords: Evolution of virulence; Coexistence; Persistence

2000MSC: 92D30, 92D25

\section{Introduction}

There is a wide range of parasites that are both horizontally and vertically transmitted (see [1-5] and the references mentioned there). We demonstrate an apparent paradox in host-parasite dynamics that becomes manifest at endemic equilibrium in simple models without latency or recovery: the ratio of horizontal to vertical transmission decreases if the coefficient of horizontal transmission is increased. This phenomenon seems counterintuitive at first glance. It has already been observed numerically for mass-action incidence and perfect vertical transmission [2]. The mathematical properties of the model (like convergence of solutions to equilibria) were already established in [6], but without addressing the relation between horizontal and vertical transmission. We present a rigorous analytic treatment of the apparent paradox that will shed more light on the underlying mechanisms (section 3). We will show that, at endemic equilibrium, the population density decreases and the proportion of infectives increases as the coefficient of horizontal transmission is increased. In a twofold way, this decreases the pool of individuals that are available for horizontal infection while vertical transmission is increased. Our analysis focuses on imperfect vertical transmission and works for arbitrary incidence functions which include mass-action and standard incidence as boundary cases. 
Since some parasites like endophytic fungi seem to rely on vertical transmission alone, we add to the model a second parasite strain (VT strain) that is completely vertically transmitted (section 4). Somewhat loosely, we call the first strain the horizontally transmitted strain (HT strain), though it can be not only horizontally but possible also vertically transmitted. We assume that there is complete cross-protection between the strains, i.e. a host that has been infected by one strain cannot be infected by the other strain. Cross-protection that is at least partially effective has been found in the woodland grass Brachypodium sylvaticum where verticle infection by the fungus Epichloë sylvatica makes plants less suseptible to infection by horizontally transmitted strains [4]. We also assume that a host that has been infected one way (horizontally or vertically) cannot be infected again by the same strain the other way (or that the second infection would have no effect). Since vertical transmission is not perfect, the vertically transmitted strain would die out if it were just by itself. We will see that, if the HT strain has a sufficiently large horizontal transmission coefficient and is more virulent than the VT strain, the two strains can coexist. The VT strain is able to persist because it protects the host against the more virulent HT strain. This has already been observed in simulations for mass-action incidence [3]; we provide an analytic proof that covers mass action and standard incidence and all interpolating incidence forms. In this paper we will show coexistence of both parasite strains (and the host) at equilibrium (section 5), while dynamic coexistence (where the densities of both strains are eventually bounded away from 0 even if they are quite low initially) will be proved in [7] using dynamical systems persistence theory [8].

The coexistence of the HT and the VT strain is of interest for parasite evolution. At carrying host capacity (in absence of the parasite), the VT strain has a replacement ratio which is strictly smaller than 1 while the HT strain has a replacement ratio which is larger than 1 . So the coexistence of the two strains is a counterexample to the principle of $\mathcal{R}_{0}$-maximization that strains with higher basic replacement ratio drive strains with lower basic replacement ratio into extinction (see [9] for a survey and references). In view of the competitive exclusion principle, there are two consumers (the two parasite strains) and one resource (the host); still the two consumers coexist because horizontal and vertical transmission offer two different routes of resource utilization.

In the two-strain model, the paradox of horizontal and vertical transmission observed in the one-strain model manifests itself in the relation between the two strains (section 5.2): at coexistence equilibrium, the ratio of the hosts infected by the HT strain to the hosts infected by the VT strain is a strictly decreasing function of the horizontal transmission coefficient. This ratio is very small, if the horizontal transmission coefficient is very large and the vertical transmission of the VT strain is almost perfect.

\section{A model with horizontal and vertical transmission}

Without the disease, the population with density $N(t)$ at time $t$ develops as

$$
N^{\prime}=(\beta(N)-\mu(N)) N
$$

where $\beta(N)$ and $\mu(N)$ are the per capita reproduction and mortality rates (of healthy individuals).

Assumption $2.1 \quad \beta(N)$ is a decreasing positive function of $N \geq 0, \mu(N)$ is an increasing positive function of $N \geq 0$. Both are continuously differentiable, $\beta^{\prime}(N)-\mu^{\prime}(N)<0$ for all $N>0 . \beta(N)-\mu(N)$ is positive for $N=0$ and negative for large $N>0$. 
It follows from these assumptions that there exists a unique number $K>0$ such that

$$
\beta(K)-\mu(K)=0 .
$$

$K$ is called the carrying capacity of the host population in absence of the disease, because $N(t) \rightarrow K$ as $t \rightarrow \infty$ provided $N(0)>0$.

The disease divides the population into a susceptible part, with density $S(t)$, and an infective part, with density $I(t)$. The infective part is again divided into those that have been horizontally infected and those that have been vertically infected, the respective densities are denoted by $I_{h}$ and $I_{v}$.

$$
\begin{aligned}
& N=S+I, \quad I=I_{h}+I_{v}, \\
& S^{\prime}=\beta(N)(S+q(1-p) I)-\mu(N) S-\frac{\sigma C(N) S I}{N}, \\
& I_{h}^{\prime}=\frac{\sigma C(N) S I}{N}-\mu(N) I_{h}-\alpha I_{h}, \\
& I_{v}^{\prime}=q p \beta(N) I-\mu(N) I_{v}-\alpha I_{v} .
\end{aligned}
$$

The infection is vertically transmitted at the probability $p \in[0,1]$. Infected individuals reproduce at the reduced rate $q \beta(N), q \in[0,1] . \alpha$ is the additional per capita rate of dying from the disease. $C(N)$ describes how the per capita rate or amount of contacts depends on the population size $N$. These may be direct contacts as in sexually transmitted diseases or indirect contacts as through spores in fungal plant diseases. The parameter $\sigma$ is a compound parameter which combines parameters describing sexual activity or spore production with the per capita infection rate or probability under the condition that a contact has been made and the contact is between a susceptible and infective individual. The precise interpretations of $C(N)$ and $\sigma$ depend on the specific transmission mechanisms of the disease. The parameter $\sigma$ will be of central importance in our analysis, and we call it the horizontal transmission coefficient.

$I / N$ is the conditional probability that a given contact made by a susceptible individual actually occurs with an infective individual. If $C(N)$ does not depend on $N$, we have the special case of standard incidence, if $C(N)$ is proportional to $N$ the special case of mass action incidence. Examples for several choices that have been made for the contact function $C$ in the literature have been collected in [10; section 19.1]). Another example, $C(N)=\zeta \ln (1+v N)$, has been suggested for diseases of insects' ([11; appendix B] and the references therein).

ASSUMPTION 2.2 All parameters are nonnegative, $q>0$ (the disease does not sterilize), $p<1$ (vertical transmission typically is imperfect).

$C(N)$ is an increasing function of $N \geq 0, C(N)>0$ for $N>0 . C(N)$ is continuously differentiable at $N>0$.

The assumptions $q>0$ and $p<1$ are not needed for the existence theorem below. We add the equations for $I_{h}$ and $I_{v}$ to obtain an equation for $I$ and also replace the equation for $S$ by an equation for $N$,

$$
\begin{aligned}
N^{\prime} & =(\beta(N)-\mu(N)) N-((1-q) \beta(N)+\alpha) I, \\
I^{\prime} & =\frac{\sigma C(N)(N-I) I}{N}+q p \beta(N) I-\mu(N) I-\alpha I .
\end{aligned}
$$

We introduce the fraction of infective individuals,

$$
f=\frac{I}{N} .
$$


By the quotient rule, $f^{\prime}=I^{\prime} / N-f N^{\prime} / N=f\left(I^{\prime} / I-N^{\prime} / N\right)$. The model takes the following form in terms of $N$ and $f$,

$$
\begin{aligned}
& N^{\prime}=N(\beta(N)-\mu(N)-((1-q) \beta(N)+\alpha) f), \\
& f^{\prime}=f([\sigma C(N)-\alpha-(1-q) \beta(N)](1-f)-q(1-p) \beta(N)) .
\end{aligned}
$$

This model has already been investigated in [6; section 3], but without the comparison of horizontal and vertical transmission.

THEOREM 2.3 For all $N_{0} \geq 0, f_{0} \in[0,1]$, there exists a unique non-negative solution of (6) with $N(0)=N_{0}$ and $f(0)=f_{0}$. This solution exists for all forward times, $f(t) \in[0,1]$ for all $t \geq 0$. Moreover $\lim \sup _{t \rightarrow \infty} N(t) \leq K$.

\section{The persistence equilibrium and the paradox}

Our system has one obvious equilibrium at which both the host and the parasite are extinct. Potentially, system (6) has three types of equilibria $(N, f)$ different from $(0,0)$ : the parasite extinction equilibrium $(K, 0)$ with $K>0$ where the host persists and the parasite is extinct, the host extinction equilibrium $\left(0, f^{\diamond}\right)$ with $f^{\diamond}>0$ where the host is extinct and the parasite persists (not in absolute density but in proportion), and the persistence equilibrium $\left(N^{*}, f^{*}\right)$ where both host and parasite persist.

We show that there is at most one persistence equilibrium. If it exists, the associated host density is a strictly decreasing function of the horizontal transmission coefficient $\sigma$ while the proportion of infectives is a strictly increasing function of $\sigma$. Paradoxically, the ratio $I_{h}^{*} / I_{v}^{*}$ which is also the ratio of horizontal to vertical transmission is a strictly decreasing function of $\sigma$.

More precisely (Theorem 3.1), we will find a threshold $\sigma_{1}>0$ such that the following holds:

Case 1 If $0 \leq \sigma \leq \sigma_{1}$, the disease dies out and the host converges to its carrying capacity, $K$.

For $\sigma>\sigma_{1}$, there are two cases to be considered. We define

$$
\mathcal{H}(N)=\frac{q \beta(N)}{\mu+\alpha} .
$$

$\mathcal{H}(N)$ is the reproduction ratio of the host at host density $N$ if all hosts are infective. Notice that $1 /(\mu+\alpha)$ is the life expectation and $q \beta$ the per capita birth rate of a host that is infective throughout its life. If $\mathcal{H}(0) \leq 1$, the parasite drives the host into extinction provided that all hosts are infective.

Case $2 \quad C(0)=0$ or $\mathcal{H}(0) \geq 1$.

Whenever $\sigma>\sigma_{1}$ there exists a unique persistence equilibrium. It attracts all solutions with $I(0)>0$.

Case $3 C(0)>0$ and $\mathcal{H}(0)<1$.

There exists some $\sigma_{2}>\sigma_{1}$ with the following property:

- If $\sigma_{1}<\sigma<\sigma_{2}$, there exists a unique persistence equilibrium. It attracts all solutions with $I(0)>0$. 
- If $\sigma \geq \sigma_{2}$, the parasite drives the host (and itself) into extinction.

In both cases, at the persistence equilibrium, the ratio $I_{h}^{*} / I_{v}^{*}$ which is also the ratio of horizontal infections to vertical infections is a strictly decreasing function of $\sigma$, of $\sigma \geq \sigma_{1}$ in Case 2 and of $\sigma \in\left(\sigma_{1}, \sigma_{2}\right)$ in Case 3 .

The condition $C(0)=0$ is plausible for fungal diseases of plants, for at low plant densities, the probability that a spore lands on a plant should be roughly proportional to the plant density. $C(0)>0$ may be appropriate for sexually transmitted diseases because sexually active individuals should be able to maintain a minimum level of contacts unless population densities are really low (and the validity of a deterministic model like ours breaks down).

\subsection{The persistence equilibrium}

At persistence equilibrium, from the second equation in (6),

$$
0=\left[\sigma^{*}-\alpha-(1-q) \beta^{*}\right]\left(1-f^{*}\right)-q(1-p) \beta^{*},
$$

where $\sigma^{*}=\sigma C\left(N^{*}\right)$ and $\beta^{*}=\beta\left(N^{*}\right)$. If vertical transmission were perfect, $p=1$, or the disease sterilizing, $q=0$, then $f^{*}=1$ would be a solution of this equation. This is excluded by Assumption 2.2. The equilibrium is biologically meaningful if $0<f^{*} \leq 1$, i.e. if

$$
\sigma^{*}>(1-p q) \beta^{*}+\alpha \text {. }
$$

We solve (8) for $\sigma^{*}$,

$$
\sigma^{*}=\frac{q(1-p) \beta^{*}}{1-f^{*}}+(1-q) \beta^{*}+\alpha .
$$

From the equilibrium equation for total population size in (6),

$$
0=\beta^{*}-\mu^{*}-f^{*}\left((1-q) \beta^{*}+\alpha\right),
$$

$\mu^{*}=\mu\left(N^{*}\right)$. We use a zero trick,

$$
\begin{aligned}
0 & =\beta^{*}-\mu^{*}+\left(1-f^{*}-1\right)\left((1-q) \beta^{*}+\alpha\right) \\
& =q \beta^{*}-\mu^{*}-\alpha+\left(1-f^{*}\right)\left((1-q) \beta^{*}+\alpha\right) .
\end{aligned}
$$

We solve this equation for $1-f^{*}$,

$$
1-f^{*}=\frac{\mu^{*}+\alpha-q \beta^{*}}{(1-q) \beta^{*}+\alpha} .
$$

Since $1-f^{*} \geq 0, \mu^{*}+\alpha-q \beta^{*} \geq 0$. We substitute (11) into (9),

$$
\begin{aligned}
\sigma C\left(N^{*}\right) & =\sigma^{*}=\frac{q(1-p) \beta^{*}\left((1-q) \beta^{*}+\alpha\right)}{\mu^{*}+\alpha-q \beta^{*}}+(1-q) \beta^{*}+\alpha \\
& =\left(\frac{q(1-p) \beta^{*}}{\mu^{*}+\alpha-q \beta^{*}}+1\right)\left((1-q) \beta^{*}+\alpha\right) .
\end{aligned}
$$

We recall that $\beta^{*}=\beta\left(N^{*}\right)$ is a decreasing and $\mu^{*}=\mu\left(N^{*}\right)$ an increasing function of $N^{*}$ and $\beta\left(N^{*}\right)-\mu\left(N^{*}\right)$ is a strictly decreasing function of $N^{*}$ (Assumption 2.1). So $\mu\left(N^{*}\right)-q \beta\left(N^{*}\right)=(1-q) \mu\left(N^{*}\right)+q\left(\beta\left(N^{*}\right)-\mu\left(N^{*}\right)\right)$ is a strictly increasing function of $N^{*}$ (Assumption 2.2). This implies that the right-hand side of (12) is a strictly decreasing 
function of $N^{*}$. Since $C$ is an increasing function, there exists at most one solution $N^{*}>0$ of this equation and $N^{*}$ depends on $\sigma$ in a strictly decreasing way. The right-hand side of (11) is a strictly increasing function of $N^{*}$ and so $f^{*}$ is a strictly increasing function of $\sigma$.

We define

$$
\mathcal{R}(N)=\frac{\sigma C(N)+q p \beta(N)}{\mu(N)+\alpha} .
$$

$\mathcal{R}(N)$ is the basic replacement ratio of the parasite at host population density $N$, i.e. the average number of new infective hosts produced by one infective host which is introduced into a completely susceptible population of density $N$. Notice that $1 /(\mu(N)+\alpha)$ is the mean length of the infective period. $\sigma C(N)$ is the average rate at which a typical infective individual produces new infections by horizontal transmission if it is introduced into a completely susceptible population of density $N . q p \beta(N)$ is the average rate at which a typical infective individual produces new infections by vertical transmission if the population density is $N$. Recall the carrying host capacity $K$ in (2) and the reproduction ratio $\mathcal{H}(N)$ of infective hosts in (7).

THEOREM 3.1 There exists at most one equilibrium $\left(N^{*}, f^{*}\right)$ at which both host and parasite persist, $N^{*}, f^{*}>0$. If the persistence equilibrium exists, $N^{*}$ depends in a strictly decreasing way on $\sigma$ while the fraction of infectives $f^{*}$ depends in a strictly increasing way on $\sigma$.

The persistence equilibrium exists, with $0<N^{*}<K$, if and only if the following two conditions are satisfied:

(a) $\mathcal{R}(K)>1$,

(b) either $\mathcal{H}(0) \geq 1$

$$
\text { or }\left\{\begin{array}{l}
\mathcal{H}(0)<1 \quad(\text { i.e. } \mu(0)+\alpha-q \beta(0)>0) \\
\sigma C(0)<\left(\frac{q(1-p) \beta(0)}{\mu(0)+\alpha-q \beta(0)}+1\right)((1-q) \beta(0)+\alpha) .
\end{array}\right.
$$

If $\mathcal{H}(0) \geq 1$, i.e. $\mu(0)+\alpha-q \beta(0) \leq 0$, then $N^{*}>\tilde{K}$ with $\mu(\tilde{K})+\alpha-q \beta(\tilde{K})=0$ whatever the value of $\sigma$.

Notice that condition (b) is satisfied if $C(0)=0$ which includes the case of mass action incidence. Condition (a) guarantees that $f^{*}>0$ while (b) guarantees that $N^{*}>0$. If (a) holds but not (b), then the disease drives the host into extinction (Theorem 3.6). The threshold parameter $\sigma_{1}$ mentioned at the beginning of this section is determined from $\mathcal{R}(0)=1$ while $\sigma=\sigma_{2}$ makes the third inequality in (b) an equality. $\sigma_{1}<\sigma_{2}$ follows from $\beta(K)=\mu(K)$ and the monotonity properties of $\beta, \mu, C$.

Proof We have already shown the monotone dependence of $N^{*}$ and $f^{*}$ on $\sigma$ and only need to discuss the existence of the persistence equilibrium.

(Necessity of (a) and (b)) By (10) and $\beta(K)=\mu(K)$ and the monotonicity assumptions for $\beta$ and $\mu$, necessarily $0<N^{*}<K$. Recall the monotonicity assumptions for $\beta, \mu$, and $C$ : replacing $N^{*}$ by $K$ in (12) makes the left hand side larger than the right-hand side. This amounts to

$$
\sigma C(K)>\left(\frac{q(1-p) \beta(K)}{\mu(K)+\alpha-q \beta(K)}+1\right)((1-q) \beta(K)+\alpha) .
$$

Since $\beta(K)=\mu(K)$, this can be rewritten in replacement ratio form (a). In order to show the necessity of (b), we can assume that $\mu(0)+\alpha-q \beta(0)>0$. Then $\mu(N)+\alpha-q \beta(N)>0$ for all $N \geq 0$ and the right-hand side of (12) is strictly decreasing in $N^{*}$. This implies (b). 
(Sufficiency of (a) and (b)) We apply the intermediate value theorem. Assume $\mu(0)+\alpha-$ $q \beta(0)>0$. Then the right-hand side of (12) is defined and positive for all $N^{*} \geq 0$, and larger than the left-hand side for $N^{*}=0$ and smaller than the left-hand side for $N^{*}=K$. So equality holds for some $N^{*} \in(0, K)$. Now assume $\mu(0)+\alpha-q \beta(0) \leq 0$. Then we can find some $\widetilde{N} \in(0, K)$ such that $\mu(\tilde{N})+\alpha-q \beta(\widetilde{N})>0$ but so close to 0 that the right hand side of (12) is larger than the left-hand side. If $\mu(0)+\alpha-q \beta(0)<0$, we can choose $\widetilde{N} \geq \widetilde{K}>0$. By the intermediate value theorem, (12) holds for some $N^{*} \in(\widetilde{N}, K)$.

\subsection{The apparent paradox}

While the decreasing dependence on $\sigma$ of the total population size at persistence equilibrium makes intuitive sense, the decreasing dependence of the subsequent ratios seems counterintuitive at first. We define the ratio of horizontally to vertically infected individuals

$$
r=\frac{I_{h}}{I_{v}}
$$

and the ratio of horizontal to vertical incidence,

$$
\rho=\frac{\sigma C(N)(S / N)}{p q \beta(N)} .
$$

The two ratios $r$ and $\rho$ are different dynamic quantities. However, at persistence equilibrium, they coincide because the last two equations in (3) imply that

$$
r^{*}=\frac{I_{h}^{*}}{I_{v}^{*}}=\frac{\sigma^{*} S^{*}}{q p \beta^{*} N^{*}} .
$$

So, at persistence equilibrium, we can generically speak about the ratio of horizontal to vertical transmission independently of whether we have a ratio of prevalences or incidences in mind. Since $S / N=(N-I) / N=1-f$,

$$
r^{*}=\frac{\sigma^{*}\left(1-f^{*}\right)}{q p \beta^{*}}
$$

We use (9) to eliminate $\sigma^{*}$,

$$
r^{*}=\frac{1-p}{p}+\left(1-q+\frac{\alpha}{\beta^{*}}\right) \frac{1-f^{*}}{p q} .
$$

The right-hand side of (17) is an increasing function of $N^{*}$ (recall $\beta^{*}=\beta\left(N^{*}\right)$ ) and a strictly decreasing function of $f^{*}$. Since $N^{*}$ is a (strictly) decreasing function of $\sigma$ and $f^{*}$ a strictly increasing function of $\sigma$ by Theorem 3.1, $r^{*}$ is a strictly decreasing function of $\sigma$.

THEOREM 3.2 The ratio $r^{*}$ of horizontal to vertical transmission at persistence equilibrium is a strictly decreasing function of the coefficient of horizontal transmission $\sigma$. If $q \beta(0)>$ $\mu(0)+\alpha$ (a mild disease) or $C(0)>0$,

$$
\left.\begin{array}{llc}
f^{*} & \longrightarrow & 1 \\
r^{*} & \longrightarrow & \frac{1-p}{p}
\end{array}\right\} \sigma \longrightarrow \infty .
$$

Proof We already proved the first statement. If $C(0)>0, \sigma^{*} \geq \sigma C(0) \rightarrow \infty$ as $\sigma \rightarrow \infty$. If $q \beta(0)>\mu(0)+\alpha$, by Theorem $3.1, N^{*}>\widetilde{K}>0$ with $q \beta(\widetilde{K})=\mu(\widetilde{K})+\alpha$ and $\sigma^{*} \geq$ $\sigma C(\widetilde{K}) \rightarrow \infty$ as $\sigma \rightarrow \infty$ as well. $f^{*} \rightarrow 1$ follows from (9) and $\beta(K) \leq \beta^{*} \leq \beta(0)$. By (17), $r^{*} \rightarrow(1-p) / p$. 


\subsection{Transient dynamics}

The proof of Theorem 3.2 shows that the apparent paradox results from the combination of two effects: as $\sigma$ is increased, the host density is decreased and the proportion of infected individuals increased. In order to emphasize that the paradox only holds at persistence equilibrium, we investigate how the ratio of horizontally to vertically infected individuals changes from the beginning of an epidemic to the moment when the persistence equilibrium is approached. To this end we derive a differential equation for $r=I_{h} / I_{v}$. By the quotient rule, $r^{\prime}=\left(I_{h}^{\prime} / I_{v}\right)-$ $r\left(I_{v}^{\prime} / I_{v}\right)$. We substitute the appropriate differential equations from (3),

$$
r^{\prime}=(1+r)(\sigma C(N)(1-f)-\operatorname{pqr} \beta(N)) .
$$

Assume that $N(0)=K$ and $f(0)$ very close to 0 . By (6) $N^{\prime}(0)$ and $f^{\prime}(0)$ are very close to 0 . This suggests that the dynamics of $N$ and $f$ are much slower than the dynamics of $r$ in the outbreak phase of the epidemic. Since $(K, 0)$ is an equilibrium, $N$ lingers quite a while close to $K$ and $f$ close to 0 , while $r$ moves to a quasi-steady state close to $\sigma C(K) /(p q \beta(K))$. But this dynamic behavior is transient and eventually $r$ converges to the steady state $\sigma C\left(N^{*}\right)\left(1-f^{*}\right) /$ $\left(p q \beta\left(N^{*}\right)\right)=r^{*}$. Notice that the transient state of the ratio $r$ is an increasing function of the horizontal transmission coefficient $\sigma$. It is much higher than the eventual equilibrium if $\sigma$ is large, mainly because $f^{*}$ is then close to 1 , but also because $N^{*}<K$ and $C(K)>C\left(N^{*}\right)$ and $\beta(K)<\beta\left(N^{*}\right)$. So we can expect a much higher ratio of horizontally to vertically infected individuals at the beginning of an epidemic than at the time when the dynamics approach the eventual equilibrium.

\subsection{Global stability of the persistence equilibrium}

We state that the persistence equilibrium, when it exists, represents the long term behavior of the host-parasite dynamics.

THEOREM 3.3 Let the assumptions ( $a$ ) and (b) of Theorem 3.1 be satisfied. Then the persistence equilibrium $\left(N^{*}, f^{*}\right)$ is locally stable and all solutions $(N, f)$ of $(6)$ with $N(0)>0$ and $f(0) \in$ $(0,1]$ satisfy $N(t) \rightarrow N^{*}$ and $f(t) \rightarrow f^{*}$ for $t \rightarrow \infty$. Further $I_{h}(t) \rightarrow I_{h}^{*}$ and $I_{v}(t) \rightarrow I_{v}^{*}$ as $t \rightarrow \infty$.

Proof The local stability of $\left(N^{*}, f^{*}\right)$ follows by linearization and a straightforward application of the Routh-Hurwitz criterion. The convergence of $f$ and $N$ is proved in [6; section 3]. By (3),

$$
\begin{aligned}
& I_{h}^{\prime}=N \sigma C(N)(1-f) f-\mu(N) I_{h}-\alpha I_{h}, \\
& I_{v}^{\prime}=q p N f \beta(N)-\mu(N) I_{v}-\alpha I_{v},
\end{aligned}
$$

and the convergence of $I_{h}$ and $I_{v}$ follows as well.

For completeness, we mention the long term behavior of the model if the persistence equilibrium does not exist. The proofs can be found in [6; section 3].

THEOREM 3.4 Assume that $\mathcal{R}(K) \leq 1$. Then $f(t) \rightarrow 0$ and $N(t) \rightarrow K$ for every solution with $N(0)>0, f(0) \in[0,1]$.

In anticipation of the upcoming two-strain discussion in the following sections, we notice that a completely vertically transmitted parasite dies out. 
COROLlaRY 3.5 A completely vertically transmitted parasite $(\sigma=0)$ dies out, unless vertical transmission is perfect, $p=1$, and the parasite is completely harmless, $q=1$ and $\alpha=0$.

Proof Let $\sigma=0$. Recall that $\beta(K)=\mu(K)$. So the sufficient condition for parasite extinction in Theorem 3.4 is satisfied if $0<(1-p q) \mu(K)+\alpha$, i.e. if $p q<1$ or $\alpha>0$.

Theorem 3.6 Assume that $\mathcal{R}(K)>1$. Let $\sigma_{0}=\sigma C(0), \beta_{0}=\beta(0)$ and $\mu_{0}=\mu(0)$ and also assume

$$
\mu_{0}+\alpha-q \beta_{0}>0 \quad \text { and } \quad \sigma_{0}>\left(\frac{q(1-p) \beta_{0}}{\mu_{0}+\alpha-q \beta_{0}}+1\right)\left((1-q) \beta_{0}+\alpha\right) .
$$

Then $N(t) \rightarrow 0$ as $t \rightarrow \infty$ and

$$
f(t) \longrightarrow \frac{\sigma_{0}-(1-q p) \beta_{0}-\alpha}{\sigma_{0}-(1-q) \beta_{0}-\alpha}
$$

for every solution $(N, f)$ of $(2.6)$ with $N(0) \geq 0$ and $f(0) \in(0,1]$.

\section{The two-strain model with one strain only vertically transmitted}

We extend our model to allow for two strains of the parasite. We assume that there is crossprotection between the strains, i.e. a host that has been infected by one strain cannot be infected by the other strain. We also assume that a host that has been infected in one way (horizontally or vertically) cannot be again infected by the same strain the other way (or that the second infection has no effect). Finally we assume that the second strain is only vertically transmitted. Somewhat imprecisely, we will speak about the first strain as the horizontally transmitted strain (HT strain) and about the second strain as the vertically transmitted strain (VT strain). We will see that, if the horizontal transmission coefficient of the HT strain is sufficiently high and the VT strain is less virulent, the two strains can coexist. We will also show that, at coexistence equilibrium, the ratio of the hosts infected by the HT strain to the hosts infected by the VT strain is a strictly decreasing function of the horizontal transmission coefficient and can be very small if the horizontal transmission coefficient is very large and the vertical transmission of the VT strain is almost perfect.

As before, $N$ denotes the total host density, $S$ the density of susceptible, uninfected, hosts, and $I_{j}$ denotes the density of hosts infected with strain $j, I_{1}$ with the HT strain and $I_{2}$ with the VT strain,

$$
\left\{\begin{array}{l}
N=S+I_{1}+I_{2} \\
S^{\prime}=\beta(N)\left(S+\sum_{k=1}^{2} q_{k}\left(1-p_{k}\right) I_{k}\right)-\mu(N) S-\frac{C(N) S}{N} \sigma I_{1} \\
I_{1}^{\prime}=I_{1}\left(\frac{C(N) S}{N} \sigma+q_{1} p_{1} \beta(N)-\mu(N)-\alpha_{1}\right) \\
I_{2}^{\prime}=I_{2}\left(q_{2} p_{2} \beta(N)-\mu(N)-\alpha_{2}\right) .
\end{array}\right.
$$

Compare system (19) with two parasite strains with the one-strain system (3) for formal analogies and differences. The parameters and parameter functions have the same meaning as before, but the epidemiologic parameters now carry an index which denotes the parasite strain. While we were interested in the ratio $I_{h} / I_{v}$ for system (3), we will investigate the ratio $I_{1} / I_{2}$ for system (19). It is also instructive to compare (19) to the system (4) which is the sub-model 
of (19) with the VT strain being absent (set $I_{2} \equiv 0$ and identify $I_{1}$ in (19) with $I$ in (4)). So all results of section 3 apply to the dynamics for (19) on the invariant boundary set $\left\{I_{2}=0\right\}$.

In the same way as for the one-strain model, we rewrite (19) in terms of the total host density,

$$
\begin{aligned}
& N^{\prime}=(\beta(N)-\mu(N)) N-\sum_{k=1}^{2}\left(\left(1-q_{k}\right) \beta(N)+\alpha_{k}\right) I_{k}, \\
& I_{1}^{\prime}=I_{1}\left(\sigma C(N) \frac{N-\sum_{k=1}^{2} I_{k}}{N}+q_{1} p_{1} \beta(N)-\mu(N)-\alpha_{1}\right), \\
& I_{2}^{\prime}=I_{2}\left(q_{2} p_{2} \beta(N)-\mu(N)-\alpha_{2}\right) .
\end{aligned}
$$

Keep in mind that $N \geq I_{1}+I_{2}$. We introduce the fraction of individuals infected by strain $j$,

$$
f_{j}=\frac{I_{j}}{N}
$$

By the quotient rule, $f_{j}^{\prime}=f_{j}\left(I_{j}^{\prime} / I_{j}-N^{\prime} / N\right)$. We rewrite the system in terms of the total host density and the fractions of strain $j$ parasites,

$$
\begin{aligned}
N^{\prime}= & N\left(\beta(N)-\mu(N)-\sum_{k=1}^{2}\left(\left(1-q_{k}\right) \beta(N)+\alpha_{k}\right) f_{k}\right), \\
f_{1}^{\prime}= & f_{1}\left(\sigma C(N)\left(1-f_{1}-f_{2}\right)-\left(1-q_{1} p_{1}\right) \beta(N)-\alpha_{1}\right. \\
& \left.+\sum_{k=1}^{2}\left(\left(1-q_{k}\right) \beta(N)+\alpha_{k}\right) f_{k}\right), \\
f_{2}^{\prime}= & f_{2}\left(-\left(1-q_{2} p_{2}\right) \beta(N)-\alpha_{2}+\sum_{k=1}^{2}\left(\left(1-q_{k}\right) \beta(N)+\alpha_{k}\right) f_{k}\right) .
\end{aligned}
$$

$\sigma$ is again called the coefficient of horizontal transmission. We keep Assumption 2.1, but replace Assumption 2.2.

AsSUMPTION 4.1 All parameters are non-negative. Both strains do some harm to the host, $\alpha_{j}>0$ or $q_{j}<1$ for $j=1,2$. However, neither strain sterilizes the host, i.e. $q_{j}>0$ for $j=1,2$. Furthermore, vertical transmission is imperfect for both strains, $p_{j}<1$ for $j=1,2$.

$C(N)$ is an increasing function of $N \geq 0, C(N)>0$ for $N>0 . C(N)$ is continuously differentiable at $N>0$.

The following result is proved in [7].

THEOREM 4.2 Let $\breve{N}$ and $\breve{f}_{1}, \breve{f}_{2}$ be non-negative numbers, $\breve{f}_{1}+\breve{f}_{2} \leq 1$. Then there exists a unique non-negative solution of (22) on $\mathbb{R}_{+}$such that $N(0)=\breve{N}, f_{j}(0)=\breve{f}_{j}$ for $j=1,2$. Moreover $f_{1}(t)+f_{2}(t) \leq 1$ for all $t \geq 0$ and $N(t) \leq \max \{\breve{N}, K\}$ where $K$ is the carrying capacity for the parasite-free host population, $\beta(K)=\mu(K)$. Finally $\lim \sup _{t \rightarrow \infty} N(t) \leq K$. 


\section{Coexistence at equilibrium}

The system (22) can have as many as six equilibria. We are interested in the equilibrium at which both strains and the host coexist. Such an equilibrium (simply called a coexistence equilibrium) is associated with an equilibrium of (20) and, from the third equation of this system,

$$
0=q_{2} p_{2} \beta\left(N^{*}\right)-\mu\left(N^{*}\right)-\alpha_{2}
$$

This equation only has a solution if $q_{2} p_{2}>0$. Since, by Assumption 2.1, $q_{2} p_{2} \beta(N)-\mu(N)$ is a strictly decreasing function of $N$, we learn that the persistence of the VT strain uniquely determines the equilibrium value of the host, $N^{*}$. This strongly suggests that the coexistence equilibrium is unique and it will turn out to be this way. We also learn that $N^{*}$ does not depend on $\sigma$ or any other parameter specifically related to the HT strain. In replacement ratio language, (23) becomes

$$
\mathcal{R}_{2}\left(N^{*}\right)=1
$$

Here

$$
\mathcal{R}_{2}(N)=\frac{q_{2} p_{2} \beta(N)}{\mu(N)+\alpha_{2}}
$$

is the basic replacement ratio of the VT strain at population density $N$ and is the special case $\sigma=0$ of (13) for the VT strain. It is also the basic reproduction ratio of a typical individual infected by the VT strain. $\mathcal{R}_{2}\left(N^{*}\right)=1$ has a solution with $N^{*}>0$ if and only if

$$
\mathcal{R}_{2}(0)>1
$$

This requires that the VT strain is not too virulent (recall $\beta(0)>\mu(0)$ ) and implies that $q_{2} p_{2}>0$.

We will discover that the existence of a coexistence equilibrium also requires the HT strain to be more virulent than the VT strain, in the following sense,

$$
\left(q_{2} p_{2}-q_{1}\right) \beta\left(N^{*}\right)+\alpha_{1}-\alpha_{2}>0 .
$$

Finally we will find a threshold value $\sigma_{b}>0$ such that the coexistence equilibrium exists if and only if $\sigma>\sigma_{b}$ and the virulence relations $\mathcal{R}_{2}(0)>1$ and (27) hold. The equilibrium values of infective hosts $I_{1}^{*}+I_{2}^{*}$ and the fraction of infective hosts $f_{1}^{*}+f_{2}^{*}$ turn out to be strictly increasing functions of $\sigma>\sigma_{\mathrm{b}}$ while the ratio $I_{1}^{*} / I_{2}^{*}=f_{1}^{*} / f_{2}^{*}$ of HT-strain infected hosts to VT-strain infected hosts is a strictly decreasing function of $\sigma$.

Comparing $\sigma_{b}$ to the threshold parameter $\sigma_{1}$ related to the existence of the one-strain persistence equilibrium in section 3, we will find that $\sigma_{b}>\sigma_{1}$ and also $\sigma_{b}<\sigma_{2}$ if Case 3 in section 3 holds.

The protection that the VT strain provides against the HT strain takes the following form. If Case 3 in section 3 holds and $\sigma \geq \sigma_{2}$, then the HT strain drives the host and itself into extinction in absence of the VT strain, while host and parasite coexist with the VT strain present. If Case 2 holds with $\sigma>\sigma_{b}$ or Case 3 with $\sigma \in\left(\sigma_{b}, \sigma_{2}\right)$, the system with only the HT strain converges to an equilibrium with a host density $N^{\sharp}$ which is less than the host density $N^{*}$ at the coexistence equilibrium. Since $N^{\sharp}$ is a strictly decreasing function of $\sigma$ and $N^{*}$ does not depend on $\sigma$, the protective effect increases with $\sigma$. 
THEOREM 5.1 A (uniquely determined) coexistence equilibrium exists if and only if the following assumptions are satisfied:

(a) $\mathcal{R}_{2}(0)>1$.

(b) The VT strain is less harmful than the HT strain in the following way,

$$
\left(q_{2} p_{2}-q_{1}\right) \beta\left(N^{*}\right)+\alpha_{1}-\alpha_{2}>0,
$$

where $N^{*}$ is the unique solution of $\mathcal{R}_{2}\left(N^{*}\right)=1$.

(c) The horizontal transmission coefficient is large enough, $\sigma>\sigma_{b}$, i.e.,

$$
\sigma C\left(N^{*}\right)>\frac{\left(q_{2} p_{2}-q_{1} p_{1}\right) \beta\left(N^{*}\right)+\alpha_{1}-\alpha_{2}}{\left(q_{2} p_{2}-q_{1}\right) \beta\left(N^{*}\right)+\alpha_{1}-\alpha_{2}}\left(\left(1-q_{1}\right) \beta\left(N^{*}\right)+\alpha_{1}\right) .
$$

The thresholds mentioned before are determined by the equation

$$
\sigma=\left(1+\frac{q_{1}\left(1-p_{1}\right) \beta(N)}{\mu(N)+\alpha_{1}-q_{1} \beta(N)}\right) \frac{\left(1-q_{1}\right) \beta(N)+\alpha_{1}}{C(N)},
$$

where $N=K$ for $\sigma=\sigma_{1}($ recall $\beta(K)=\mu(K)), N=0$ for $\sigma=\sigma_{2}($ if $C(0)>0)$, and $\sigma=\sigma_{b}$ for $N=N^{*}$ (recall (23)). Since $0<N^{*}<K$, our monotonicity assumptions imply $\sigma_{1}<\sigma_{b}$ and $\sigma_{b}<\sigma_{2}$ if $\sigma_{2}$ is defined.

Proof We already discussed that the existence of a solution $N^{*}>0$ of $\mathcal{R}_{2}\left(N^{*}\right)=1$ is equivalent to (a). Since $N^{*}$ is uniquely determined by this equation, we suppress it as an argument of $C, \beta$, and $\mu$ in the subsequent algebraic manipulations. An equilibrium where both parasite strains and the host coexist is also an equilibrium of system (20). Its second equation can be rewritten as

$$
I_{1}^{\prime}=I_{1}\left(\sigma C(N)(1-\bar{f})+q_{1} p_{1} \beta(N)-\mu(N)-\alpha_{1}\right),
$$

with $\bar{f}=f_{1}+f_{2}$ denoting the fraction of all infective individuals. At the coexistence equilibrium,

$$
0=\sigma C\left(1-\bar{f}^{*}\right)+q_{1} p_{1} \beta-\mu-\alpha_{1} .
$$

We subtract the equations in (30) and (23),

$$
\sigma C\left(1-\bar{f}^{*}\right)=\alpha_{1}-\alpha_{2}+\left(q_{2} p_{2}-q_{1} p_{1}\right) \beta .
$$

We solve this equation for $1-\bar{f}^{*}$

$$
1-\bar{f}^{*}=\frac{\alpha_{1}-\alpha_{2}+\left(q_{2} p_{2}-q_{1} p_{1}\right) \beta}{\sigma C} .
$$

The equation for $f_{2}$ in (22) at coexistence equilibrium is

$$
0=-\left(1-q_{2} p_{2}\right) \beta-\alpha_{2}+\sum_{k=1}^{2} f_{k}^{*}\left(\left(1-q_{k}\right) \beta+\alpha_{k}\right) .
$$

We solve this equation for $f_{1}^{*}$ in terms of $\bar{f}^{*}=f_{1}^{*}+f_{2}^{*}$,

$$
f_{1}^{*}=\frac{q_{2}\left(1-p_{2}\right) \beta+\left(\left(1-q_{2}\right) \beta+\alpha_{2}\right)\left(1-\bar{f}^{*}\right)}{\left(q_{2}-q_{1}\right) \beta+\alpha_{1}-\alpha_{2}} .
$$

We obtain meaningful expressions if and only if $0<f_{1}^{*}<\bar{f}^{*} \leq 1$. Actually, by (33), $f_{1}^{*}>0$ if and only if $\bar{f}^{*}<1$. 
By (32), (b) is necessary for $\bar{f}^{*}<1$ (recall that $p_{1}\left(1-q_{1}\right)>0$ ) while (b) and (c) are sufficient. Notice that (23) implies $\left(q_{2} p_{2}-1\right) \beta\left(N^{*}\right)-\alpha_{2}<0$. The inequality in (b) guarantees that the denominator in (33) is positive. So (b) and (c) imply that $\bar{f}^{*}<1$ and $f_{1}^{*}>0$.

By (33), $f_{1}^{*}<\bar{f}^{*}$ is equivalent to

$$
\bar{f}^{*}\left(\left(q_{2}-q_{1}\right) \beta+\alpha_{1}-\alpha_{2}\right)>q_{2}\left(1-p_{2}\right) \beta+\left(\left(1-q_{2}\right) \beta+\alpha_{2}\right)\left(1-\bar{f}^{*}\right) .
$$

We solve this inequality for $1-\bar{f}^{*}$,

$$
1-\bar{f}^{*}<\frac{\left(q_{2} p_{2}-q_{1}\right) \beta+\alpha_{1}-\alpha_{2}}{\left(1-q_{1}\right) \beta+\alpha_{1}} .
$$

This inequality can only be satisfied for some $\bar{f}^{*} \in(0,1)$ if the inequality in (b) holds. Substituting (32), we see that (34) holds if and only if the inequality in (c) holds. $\bar{f}^{*}>0$ now follows from $\bar{f}^{*}>f_{1}^{*}>0$.

Remark 5.2 The condition in Theorem 5.1(b) is equivalent to

$$
\mu\left(N^{*}\right)+\alpha_{1}-q_{1} \beta\left(N^{*}\right)>0 .
$$

The condition in Theorem 5.1(c) is equivalent to

$$
1>\left(1+\frac{q_{1}\left(1-p_{1}\right) \beta\left(N^{*}\right)}{\mu\left(N^{*}\right)+\alpha_{1}-q_{1} \beta\left(N^{*}\right)}\right) \frac{\left(1-q_{1}\right) \beta\left(N^{*}\right)+\alpha_{1}}{\sigma C\left(N^{*}\right)} .
$$

Notice that the right-hand side of this equation is strictly decreasing. So the condition in Theorem 5.1(c) implies that

$$
\sigma C(K)>\left(1-q_{1} p_{1}\right) \beta(K)+\alpha_{1}
$$

which, by $\beta(K)=\mu(K)$, is equivalent to condition (a) in Theorem 3.1 for $q=q_{1}, p=p_{1}$. Let us assume that condition (b) in Theorem 3.1 also holds. Then we have a boundary equilibrium $\left(\mathbb{N}^{\sharp}, f^{\sharp}, 0\right)$ where only the horizontally transmitted strain is present. By (12),

$$
1=\left(1+\frac{q_{1}\left(1-p_{1}\right) \beta\left(N^{\sharp}\right)}{\mu\left(N^{\sharp}\right)+\alpha_{1}-q_{1} \beta\left(N^{\sharp}\right)}\right) \frac{\left(1-q_{1}\right) \beta\left(N^{\sharp}\right)+\alpha_{1}}{\sigma C\left(N^{\sharp}\right)} .
$$

Since the right-hand side of this equation is decreasing, $N^{\sharp}<N^{*}$.

The question of global or even local stability of the coexistence equilibrium is not easy to address as is not unusual for models in three or higher dimensions (but see [12] and the references therein). The Jacobian determinant of the vector field is negative, essentially restricting bifurcations to be of Hopf type which is consistent with the uniqueness of the coexistence equilibrium. Since the epidemic model has an $S \rightarrow I$ structure, it is hard to see any mechanism which would produce undamped oscillations (differently from the three-strain influenza model with partial cross-immunity in [13] which has an $S \rightarrow I \rightarrow S$ structure with reduced susceptibility after recovery). However, numerical simulations suggest that undamped oscillations can occur for standard incidence and appropriate parameters. If $\sigma$ is large enough, the expressions in the Routh-Hurwitz criterion can easily be organized in an intelligible way and the following weaker result can be proved.

THEOREM 5.3 If conditions (a) and (b) in Theorem 5.1 hold and the horizontal transmission coefficient $\sigma$ is large enough, the coexistence equilibrium exists and is locally asymptotically stable.

The local stability of the coexistence can also be shown if the per capita birth rate $\beta$ does not depend on the host density $N$. 


\subsection{Dynamic coexistence}

The sufficient (and necessary) conditions for coexistence of both parasite strains and the host at equilibrium also guarantee dynamic coexistence.

THEOREM 5.4 The following are equivalent:

(i) There exists a coexistence equilibrium.

(ii) The horizontally and vertically transmitted strains coexist in the sense that there exists some $\epsilon>0$ such that

$$
\liminf _{t \rightarrow \infty} I_{j}(t) \geq \epsilon, \quad j=1,2,
$$

for all solutions of $(20)$ with $I_{1}(0)>0, I_{2}(0)>0, N(0) \geq I_{1}(0)+I_{2}(0)$.

Existence of a coexistence equilibrium is necessary for the dynamic coexistence in (b) due to a general result [14; Theorem 1.3.7]. The sufficiency is shown in [6].

A global stability result can be shown if $C$ and $\beta$ do not depend on the population density $N$.

THEOREM 5.5 [7] Assume that $C$ and $\beta$ are positive constants and that the coexistence equilibrium $x^{*}=\left(N^{*}, I_{1}^{*}, I_{2}^{*}\right)$ with $N^{*}, I_{1}^{*}, I_{2}^{*}>0$ exists. Then all solutions of (20) with $I_{1}(0), I_{2}(0)>0, N(0) \geq I_{1}(0)+I_{2}(0)$ converge towards the coexistence equilibrium.

\subsection{The paradox for the two-strain model}

How does, at endemic equilibrium, the ratio of HT-strain-infected hosts to VT-strain-infected hosts depend on the horizontal transmission coefficient $\sigma$ ?

It follows from (31) that

$$
\sigma C\left(1-\bar{f}^{*}\right)=\alpha_{1}-\alpha_{2}+\left(q_{2} p_{2}-q_{1} p_{1}\right) \beta .
$$

This implies that $\bar{f}^{*}$ is a strictly increasing function of $\sigma$. Further, as $\sigma \rightarrow \infty, \bar{f}^{*} \rightarrow 1$. Then $f_{1}^{*}$ is a strictly decreasing function of $\sigma$ by (33). Dividing equation (33) by $\bar{f}^{*}$ shows that $f_{1}^{*} / \bar{f}^{*}$ is a strictly decreasing function of $\sigma^{*}$. Since

$$
\frac{f_{1}^{*}}{f_{2}^{*}}=\frac{f_{1}^{*} / \bar{f}^{*}}{1-\left(f_{1}^{*} / \bar{f}^{*}\right)},
$$

also $f_{1}^{*} / f_{2}^{*}=I_{1}^{*} / I_{2}^{*}$ is a strictly decreasing function of $\sigma$.

THEOREM 5.6 At coexistence equilibrium, we have the following monotonicity relations with respect to the horizontal transmission coefficient $\sigma$ :

- The fraction of infected hosts, $\bar{f}^{*}$, is a strictly increasing function of $\sigma$ and $\bar{f}^{*} \rightarrow 1$ as $\sigma \rightarrow \infty$.

- The fraction of hosts infected by the HT strain, $f_{1}^{*}$, is a strictly decreasing function of $\sigma$ and so is the ratio of the hosts infected by the HT strain to the hosts infected by the VT strain, $I_{1}^{*} / I_{2}^{*}$. Moveover,

$$
f_{1}^{*} \rightarrow \frac{q_{2}\left(1-p_{2}\right) \beta\left(N^{*}\right)}{\left(q_{2}-q_{1}\right) \beta\left(N^{*}\right)+\alpha_{1}-\alpha_{2}} \quad \text { as } \sigma \rightarrow \infty .
$$




\section{Discussion}

Computational studies of host-parasite models without latency and recovery have found that the ratio of horizontal to vertical transmission decreases if the coefficient of horizontal transmission is increased [2] and that a completely vertically transmitted parasite strain that would go extinct on its own can persist in coexistence with a horizontally transmitted strain [3]. In order to understand these phenomena which may appear counterintuitive or even paradoxical, we present a rigorous analytic treatment which sheds more light on the underlying mechanisms.

The coefficient of horizontal transmission, denoted by $\sigma$, is a compound parameter whose exact interpretation depends on the specific transmission mode of the parasite. In fungal plant diseases, $\sigma$ factors in the average spore production of a typical infected plant and the conditional probability that an infection occurs once a spore has landed on a susceptible plant. In sexually transmitted diseases, $\sigma$ combines the average sexual activity of a typical sexually active person and the conditional probability that a given sexual contact between a susceptible and an infective individual actually leads to an infection.

Another important ingredient of epidemic models is the contact function $C(N)$ which describes how the per capita amount or rate of contacts depend on the host population density $N$. These may be direct contacts as in sexually transmitted diseases, or indirect contacts as through spores in fungal plant diseases. Again the precise interpretation depends on the type of disease. In fungal plant diseases, $C(N)$ is proportional to the probability at which a given spore lands on host plants rather than on the soil (or somewhere else where it is wasted) provided that the host plant density is $N$. At low host plant densities, this probability should be roughly proportional to the plant density which suggests that $C(0)=0$. In sexually transmitted diseases, $C(N)$ is proportional to the number of sexual contacts a typical sexually active person makes in a population with density $N$. Some models assume that $C(N)$ is basically independent of $N$ unless the population density is so low that a deterministic model like ours is not valid anyway. This assumption results in what is sometimes called standard incidence $[15 ; 2.1]$ and is a special case of assuming $C(0)>0$. The studies in [2,3] assume mass action incidence, where $C(N)$ is proportional to $N$ such that $C(N) / N$ does not depend on host density. Our analysis includes both standard and mass action incidence and all reasonable interpolations between these two extremes. A collection of contact functions that have been used in the literature can be found in [10; section 19.1]; another example, $C(N)=\zeta \ln (a+v N)$, has been suggested for insect diseases [11; Appendix B].

The classification of the dynamic behaviors of mathematical models typically involves thresholds for important parameters. In host-parasite models, classifications are often made in terms of the basic replacement ratio (or basic reproduction number) of the parasite as in our Theorems 3.1 and 5.1. For the purposes of this discussion, a classification according to various thresholds for the coefficient $\sigma$ of horizontal transmission may offer more insights.

In the first model which features a host and a horizontally and vertically transmitted parasite (one strain only), there exists a threshold $\sigma_{1}>0$ with the following properties:

Case 1 If $0 \leq \sigma \leq \sigma_{1}$, the parasite dies out and the host converges to its carrying capacity.

If $\sigma>\sigma_{1}$, we need to distinguish between mass action and standard incidence, more generally between the cases $C(0)=0$ and $C(0)>0$. Here another threshold parameter comes into play, $\mathcal{H}(0)$, the host reproduction ratio taken in the following scenario:

- the host population density is almost zero such that resources do not need to be shared,

- all hosts are infective. 
In the following, by definition, a persistence equilibrium is an equilibrium at which both the host and the parasite are present.

Case $2 C(0)=0$ or $\mathcal{H}(0) \geq 1$.

Whenever $\sigma>\sigma_{1}$ there exists a unique persistence equilibrium.

Case $3 C(0)>0$ and $\mathcal{H}(0)<1$.

There exists a second threshold parameter $\sigma_{2}>\sigma_{1}$ with the following property:

- If $\sigma_{1}<\sigma<\sigma_{2}$, there exists a unique persistence equilibrium.

- If $\sigma \geq \sigma_{2}$, the parasite drives the host (and itself) into extinction.

Whenever the persistence equilibrium exists, it attracts the host-parasite dynamics if both host and parasite are present initially [6]. The trichotomy formed by these three cases is not intrinsically linked to the interplay of horizontal and vertical transmission as it would also hold if vertical transmission were absent.

Our interest is in the ratio of horizontal to vertical transmission, $r$. This ratio can either be interpreted as the ratio of horizontally to vertically infected hosts (i.e. of horizontal to vertical prevalence) or the ratio of horizontal to vertical incidence. These two ratios are different dynamic quantities but coincide at persistence equilibrium.

In section 3.2 we show that, at persistence equilibrium, the ratio $r$ of horizontal to vertical transmission decreases if the horizontal transmission coefficient $\sigma$ is increased. The admissible range of $\sigma$ is $\sigma>\sigma_{1}$ in Case 2 and $\sigma_{1}<\sigma<\sigma_{2}$ in Case 3 .

In order to explain this apparent paradox one must emphasize that it holds at persistence equilibrium. In an outbreak phase of the disease, we have transient dynamics where the dependance of $r$ (here interpreted as ratio of horizontally to vertically infected hosts) on $\sigma$ is as one expects: it increases as $\sigma$ is increased (section 3.3). Our analysis shows that, at persistence equilibrium, the host population density decreases and the fraction of infected individuals increases as $\sigma$ is increased. In a twofold way, this decreases the pool of individuals which are available for horizontal infection and has the consequence that, at persistence equilibrium and for large $\sigma$, new infections are essentially driven by vertical transmission. This is of course due to our assumption that individuals infected one way can no longer be infected the other way (or that a second infection would be inconsequential). If the coefficient of horizontal transmission is very large and the disease mild enough for the host population to survive even if everyone were infected, then the ratio of horizontal to vertical transmission at endemic equilibrium is approximately $(1-p) / p$, where $p$ is the probability of vertical transmission.

Since certain endophytic fungi seem to rely on vertical transmission alone, we add to our model a second strain which is completely vertically transmitted (section 4). We refer to this second strain as VT strain while we refer to the first strain as HT strain though this strain, as before, may be both horizontally and vertically transmitted. The original model is a sub-model of this two-strain model, namely for the case that the VT strain is absent. In particular, we still have the three cases for the system consisting of the host and the HT strain. The subsystem with only the VT strain present is also a special case of the first model namely when there is no horizontal transmission (Case 1 with $\sigma=0$ ). So a parasitic strain (a strain which does not confer some benefit to its host) which is completely vertically transmitted cannot persist on its own. However, it can persist in coexistence with a more virulent HT strain (which does much more harm to the host) provided that the coefficient of horizontal transmission is large enough (section 5). More precisely, there is another threshold parameter $\sigma_{b}>0$ such that a (unique) coexistence equilibrium exists if and only if $\sigma>\sigma_{b}$. By definition, at a coexistence equilibrium the host and both parasite strains are present. $\sigma_{b}$ is related to the previous thresholds by $\sigma_{b}>\sigma_{1}$ 
and, in Case 3, by $\sigma_{1}<\sigma_{b}<\sigma_{2}$. Obviously the VT strain which would die out on its own persists because it protects the host against the more harmful HT strain. This protection can be more or less dramatic. In Case 3, if in addition $\sigma \geq \sigma_{2}$, the HT strain would drive the host and itself into extinction in the absence of the VT strain. With the VT strain around, the host and both strains coexist. In Case 3, if in addition $\sigma_{b}<\sigma<\sigma_{2}$, or in Case 2, if additionally $\sigma>\sigma_{b}$, the host and the HT strain both persist whether or not the VT strain is around, but the host has a higher density in the presence of the VT strain.

The apparent paradox between horizontal and vertical transmission can also be found in the two-strain model, though for the ratio of HT-strain-infected hosts to VT-strain-infected hosts rather than the ratio of horizontal to vertical transmission (section 5.2). At coexistence equilibrium, this ratio decreases as the coefficient of horizontal transmission (for the HT strain) is increased.

In this paper, coexistence of the host and both parasite strains is established at equilibrium while dynamic coexistence is shown in [7] using dynamical systems persistence theory. The coexistence equilibrium is locally asymptotically stable provided that the coefficient of horizontal transmission is sufficiently large. Global asymptotic stability of the coexistence equilibrium is shown in [7] for standard incidence (density-independent contact function) and a density-independent per capita birth rate.

\section{Conclusion}

This study was originally motivated by the observation that endophytic fungi from the genus Neotyphodium in Arizona grass Festuca arizonica [16-19] appear to be completely vertically transmitted via infected seeds. The fungus uses host resources for its own growth and propagation and experiments show that infected plants perform and compete worse than uninfected ones [17], and this seems to be indeed the case for most scenarios. No compensatory benefits such as those commonly found in agronomic grasses (like protection against herbivores or resistance to abiotic stresses such as drought and flooding) have been detected until very recently. Since vertical transmission is not perfect (some seeds of infected grass do not carry the fungus), it has been a mystery how the fungus persists.

Our preceding analysis suggests the possibility that horizontal transmission which could make up for imperfect vertical transmission can be easily overlooked, once the fungal disease has established itself and if vertical infection protects against horizontal infection. Paradoxically, horizontal transmission is the harder to detect if the coefficient of horizontal transmission is large than if it is small.

The one-strain model explains why horizontal transmission could be overlooked in the field, while the two-strain model explains how it may be overlooked in the laboratory as well, as chances are that host samples that have been collected in the field all happen to be infected by the vertically transmitted strain.

Of course, confirmation of this hypothesis hinges on the eventual detection of a horizontally transmitting strain which, at this point and in spite of all efforts, remains elusive.

In past studies, S.H.F. and his collaborators $[16,17]$ had found that infected plants are more attractive to herbivores than susceptible plants. Recently they also discovered that increased 'pruning' by herbivores increases allocation of host resources to seed production to the effect that infected plants produce more seeds than uninfected plants under herbivory. This amounts to $q>1$ in our first model (section 2) where $q$ is the factor by which the successful seed production and germination of infected plants changes as compared to uninfected plants. While the global stability of the model dynamics needs further investigation if $q>1$ (the Bendixson-Dulac argument in [6; Theorem 3.5] relies on $q \leq 1$ ), the condition $\mathcal{R}(K)>1$ 
is still sufficient and necessary for the persistence of the endophyte. In the case of complete vertical transmission (set $\sigma=0$ in (13)), this condition specializes to

$$
\frac{q p \beta(K)}{\mu(K)+\alpha}>1
$$

While, in this form, the condition has a suggestive interpretation in terms of the replacement ratio of the endophyte, it may not be so easy to evaluate. Since $\beta(K)=\mu(K)$, we can rewrite it as

$$
q p>\frac{L_{s}}{L_{i}}
$$

where $L_{s}$ is the life expectation of an uninfected, susceptible, plant and $L_{i}$ is the life expectation of an infected plant and $p$ the probability of vertical transmission. These life expectations must be taken, however, at the carrying plant capacity $K$ in absence of the endophyte.

\section{Acknowledgements}

This study was partially supported by NSF grants DEB 0128343 (S.H.F.), DMS 0502349 (K.P.H.) and DMS 0314529 (H.R.T.), NSA grant H98230-06-1-0097 (K.P.H.) and ASU-Sloan National Pipeline Program in Mathematical Sciences (K.P.H.).

\section{References}

[1] Busenberg, S.N. and Cooke, K.L., 1993, Vertically Transmitted Diseases: Models and Dynamics (Berlin: Springer).

[2] Lipsitch, M., Nowak, M.A., Ebert, D. and May, R.M., 1995, The population dynamics of vertically and horizontally transmitted diseases. Proceedings of the Royal Society London, B 260, 321-327.

[3] Lipsitch, M., Siller, S. and Nowak, M.A., 1996, The evolution of virulence in pathogens with vertical and horizontal transmission. Evolution, 50, 1729-1741.

[4] Meijer, G. and Leuchtmann, A., 2000, The effects of genetic and environmntal factors on disease expression (stroma formation) and plant growth in Brachypodium sylvaticum infected by Epichloë sylvatica, Oikos, 91, 446-458.

[5] Saikkonen, K., Wäli, P., Helander, M., and Faeth, S.H., 2004, Evolution of latency in foliar fungi. Trends in Plant Science, 9, 275-280.

[6] Zhou, J., and Hethcote, H.W., 1994, Population size dependent incidence in models for diseases without immunity. Journal of Mathematical Biology, 32, 809-834.

[7] Dhirasakdanon, T. and Thieme, H.R., Persistence of vertically transmitted parasite strains which protect against more virulent horizontally transmitted strains, preprint.

[8] Thieme, H.R., 1993, Persistence under relaxed point-dissipativity (with application to an epidemic model). SIAM Journal of Mathematical Analysis, 24, 407-435.

[9] Thieme, H.R., 2007, Pathogen competition and coexistence and the evolution of virulence. Mathematics for Life Sciences and Medicine, Springer, Berlin Heidelberg.

[10] Thieme, H.R., Mathematics in Population Biology (Princeton: Princeton University Press).

[11] Briggs, C.J. and Godfray, H.C.J., 1995, The dynamics of insect-pathogen interactions in stage-structured populations. The American Naturalist, 145, 855-887.

[12] Korobeinikov, A., 2006, Lyapunov functions and global stability for SIR and SIRS epidemiological models with non-linear transmission. Bulletin of Mathematical Biology, 68, 615-626.

[13] Lin, J., Andreasen, V. and Levin, S.A., 1999, Dynamics of influenza A drift: the linear three-strain model. Mathematics and Bioscience, 162, 33-51.

[14] Zhao, X.-Q., 2003, Dynamical Systems in Population Biology (New York: Springer).

[15] Hethcote, H.W., 2000, The mathematics of infectious diseases. Siam Review, 42, 599-653.

[16] Faeth, S.H., 2002, Are endophytic fungi defensive plant mutualists? Oikos, 98, 25-36.

[17] Faeth, S.H. and Bultman, T.L., 2002, Endophytic fungi and interactions among host plants, herbivores, and natural enemies. In: T. Tscharntke, and B.A. Hawkins (Eds) Multitrophic Level Interactions (Cambridge: Cambridge University Press), pp. 89-123 .

[18] Faeth, S.H. and Sullivan, T.J., 2003, Mutualistic asexual endophytes in a native grass are usually parasitic. The American Naturalist, 161, 310-325.

[19] Schulthess, F.M. and Faeth, S.H., 1998, Distribution, abundances, and associations of the endophytic fungal community of Arizona fescue (Festucca arizonica). Mycologia, 90, 569-578. 